\title{
Cultura española en la pantalla china: la transferencia de referentes culturales de la serie de televisión española El Ministerio del Tiempo en el fansubbing de China
}

\section{Spanish Culture on the Chinese Screen: The Rendering of Cultural References in the Spanish TV Series The Ministry of Time in Chinese Fansubbing}

\section{Peng GaO \\ Universitat Pompeu Fabra de Barcelona peng.gao@upf.edu}

Recibido: febrero-2020. Revisado: abril-2020. Aceptado: mayo-2020.

Resumen: El objetivo de este artículo es investigar las técnicas de traducción utilizadas por dos grupos de fansubbing de China (ShenYing y YYeTs) en la transferencia de referentes culturales de la serie de televisión El Ministerio del Tiempo. Los resultados muestran que ambos grupos tienen las mismas preferencias en técnicas de traducción a excepción de las notas en la pantalla (amplificación). Partiendo del análisis de cada técnica y su vínculo con la clasificación de referentes culturales que proponemos, se observa que, en general, la transculturalidad y los dominios a los que pertenecen influyen más que la centralidad del referente en la transferencia de los referentes verbales. En cambio, no se nota la prelación de ninguno de los tres factores en los referentes no verbales. la transferencia de referentes culturales... 
Palabras clave: traducción audiovisual; traducción español-chino; fansubbing; referentes culturales; técnicas de traducción; series de televisión españolas.

\begin{abstract}
This article aims to investigate the translation techniques used by two Chinese fansubbing groups (ShenYing and YYeTs) in the rendering of cultural references from the TV series The Ministry of Time (El Ministerio del Tiempo). The results show that both groups have the same preference in translation techniques except for notes on the screen (amplification). Based on the analysis of each technique and its connection with our classification of cultural references, we find that generally transculturality and the domains to which references are ascribed have more influence than the centrality of the reference in the rendering of verbal references, whereas, in non-verbal references, no priority of any of these three factors is found.
\end{abstract}

Keywords: audiovisual translation; translation Spanish - Chinese; fansubbing; cultural references; translation techniques; Spanish TV series.

\title{
1. INTRODUCCIÓN
}

Como indican varios estudiosos en los Estudios de Traducción como, por ejemplo, Pym (2000) y Pérez-González y Susam-Saraeva (2012), los traductores e intérpretes no profesionales han tenido, y siguen teniendo, un papel importante en el intercambio cultural a lo largo de la historia, por lo que merecen un mayor interés de la investigación. En la subtitulación, esta traducción no profesional se denomina fansubbing, sus productos fansubs y sus participantes fansubbers (Ferrer Simó 2005; Díaz-Cintas y Muñoz Sánchez 2006).

El fansubbing emergió en China en 2001 (Tian 2011). Al principio se trataba sobre todo de fansubbers que, a título individual, se dedicaban a la subtitulación del anime o de los videojuegos. Aproximadamente en 2002 se formaron los primeros grupos de fansubbing del anime. Después de ellos, fueron surgiendo grupos de fansubbing de series y películas japonesas y coreanas. En 2003 se estableció F6, el primer foro en línea sobre la serie de televisión estadounidense Friends (YYeTs 2009; Lee 2018). Este foro también fue el primer grupo de fansubbing de series estadounidenses en China. Con el tiempo, los fansubbers se han convertido en una comunidad importante: hasta 2017 los tres grupos más grandes en China (YYeTs, YTET y ShenYing) habían subtitulado un total de 19.230 productos de 10 lenguas (Cui 2017).

La aparición del fansubbing en China se debe a varias razones: la limitación en la importación de series y películas extranjeras (Casas-Tost y Cui 2019, 12), la censura estricta (Wang y Zhang 2016) y la preferencia de muchos jóvenes por los fansubs (LV y Li 2015, 123), entre otros. 
El fansubbing desempeña un papel esencial en la traducción del español al chino, porque relativamente pocas películas y series de televisión españolas se exportan por vías oficiales a China. De este modo, el fansubbing permite al público chino tener un mayor acceso al mundo audiovisual español y apreciar así la belleza de una cultura distinta. A pesar de la importancia del fansubbing del español al chino, este tema se ha estudiado relativamente poco hasta este momento. Entre los pocos estudios existentes, se encuentran la descripción de la estructura y la cooperación del grupo de fansubbers The Burrow (Zhang y Cassany 2016); el estudio sobre el fenómeno danmu ${ }^{1}$ a partir de los fansubs de la serie de televisión El Ministerio del Tiempo (Zhang y Cassany 2019) y el análisis contrastivo entre la versión profesional y el fansub de la serie El tiempo entre costuras (Casas-Tost y Cui 2019).

En el fansubbing del español al chino, los referentes culturales son un problema de traducción inevitable, debido a que muchos de ellos carecen de traducción oficial o equivalente establecido en la cultura receptora y, además, es posible que sean totalmente desconocidos para el público chino. A la dificultad inherente a la traducción de referentes culturales hay que añadir el espacio limitado de la pantalla. Por lo tanto, este estudio descriptivo versa sobre las técnicas de traducción en la transferencia de referentes culturales en el fansubbing del español al chino. Se basa en un corpus contrastivo construido a partir de 10 episodios de dos versiones de fansubs de la serie El Ministerio del Tiempo, en la cual encontramos abundantes referentes culturales.

\section{MARCO TEÓRICO}

En el presente estudio se utilizan los términos «referentes culturales» (Santamaria 2001, 237) para los elementos culturales y «técnicas de traducción» (Hurtado Albir 2001, 249) para referirse a los métodos utilizados por los fansubbers en la transferencia de estos elementos. A continuación, se presentará la propuesta usada para la clasificación de referentes culturales y técnicas de traducción.

\subsection{Clasificación de referentes culturales}

En nuestro estudio, los referentes culturales se clasifican de acuerdo con los dominios a los que pertenecen (Pedersen 2011; Santamaria 2001), los parámetros de influencia de transculturalidad y de centralidad de la referencia (Pedersen 2011) y los canales en textos audiovisuales (Zabalbeascoa 2001; Ranzato 2013).

1. Los danmu son comentarios del espectador sobre fotogramas determinados y recorren la pantalla de derecha a izquierda. Véase Zhang y Cassany $(2019,20)$ para más información.

Cultura española en la pantalla china: la transferencia de referentes culturales...
CLINA

vol. 6-1, June 2020, 89-108

eISSN: 2444-1961

Ediciones Universidad de Salamanca - CC BY-NC-ND 
En primer lugar, se determinan los dominios: antropónimos, topónimos, gobierno, literatura, bellas artes, etc. Esta aproximación es utilizada por numerosos autores, como, por ejemplo, Nedergaard-Larsen (1993). Véase la tabla 2 para los dominios encontrados en el corpus y sus ejemplos. En segundo lugar, se sitúa la transculturalidad, que se refiere a la familiaridad de los espectadores con respecto al TO y al TM. Según el nivel alto o bajo de este parámetro se subdividen los referentes en transculturales, monoculturales e infraculturales. Los referentes transculturales son conocidos tanto en la cultura origen como en la cultura meta. No solo pueden ser referentes derivados de la cultura origen, sino que también pueden ser los referentes de una tercera cultura. Los referentes monoculturales, en cambio, son menos conocidos para el espectador meta que para el espectador origen; y, por último, los referentes infraculturales son elementos muy específicos de la cultura origen y ni siquiera son identificados por el espectador meta (Pedersen 2011, 106-109). En tercer lugar, se contempla la centralidad del referente cultural, que evalúa la influencia del mismo sobre el argumento. Si el referente es un tema principal de un programa, se considera central a nivel macrotextual. Si el referente está relacionado con algún diálogo o con elementos de humor, es secundario a nivel macro-, pero central a nivel microtextual. Si el referente no influye en el argumento de la serie, es secundario tanto a nivel micro- como macrotextual (Pedersen 2011, 111-113). Como el argumento va variando a lo largo de los episodios, la centralidad del mismo referente puede cambiar en diferentes episodios. El último elemento de nuestra clasificación lo constituyen los canales en los textos audiovisuales. Según el canal verbal y no verbal, los referentes se clasifican como referentes verbales y referentes no verbales. Los referentes no verbales pueden ser visuales y/o acústicos (Ranzato 2013, 89).

\subsection{Clasificación de técnicas de traducción}

El presente artículo propone once técnicas de traducción adaptando las taxonomías de Pedersen (2011, 71-100), Molina (2006, 101-104) y Díaz-Cintas y Remael $(2007,206)$ para la transferencia de referentes culturales en el fansubbing del español al chino.

1) Préstamo: esta categoría se subdivide en préstamos completos, préstamos normalizados y préstamos no normalizados. El préstamo completo consiste en integrar una palabra de otra lengua sin ningún cambio. En los fansubs estudiados en el presente estudio, el préstamo completo se usa en la traducción de marcas comerciales o acontecimientos contemporáneos como el 11-M. Por las diferencias entre el español y el chino, los topónimos, los antropónimos y otros referentes culturales como los nombres de tripulaciones se integran en chino con algún cambio lingüístico obligatorio. Si este cambio se realiza de acuerdo con el estándar nacional y las recomendaciones de la Oficina de Traducción de Nombres de la Agencia

Peng $\mathrm{G}_{A O}$

Cultura española en la pantalla china: la transferencia de referentes culturales...
CLINA

vol. 6-1, June 2020, 89-108

elSSN: 2444-1961

Ediciones Universidad de Salamanca - CC BY-NC-ND 
de Noticias Xinhua, en nuestro trabajo lo hemos considerado como un préstamo normalizado; si no, se trata como un préstamo no normalizado.

2) Equivalente: incluye los equivalentes oficiales y los equivalentes acuñados (Molina 2006). El equivalente oficial se usa en la traducción de nombres propios y ha sido decidido o reconocido por alguna autoridad, como la Agencia de Noticias Xinhua, o la versión en chino del sitio web oficial del turismo de España. Un equivalente oficial puede partir de otras técnicas, como el préstamo o la traducción literal. A diferencia del equivalente oficial, el equivalente acuñado contiene una palabra, es un nombre común y es reconocido por diccionarios bilingües muy utilizados del español al chino, como el Nuevo diccionario español-chino y el Nueva era: Gran diccionario español-chino. Podemos citar como ejemplo la traducción de «pecado» por su equivalente según los diccionarios: 罪 / 罪擘 (zuì / zuiniè, crimen / pecado). En cambio, sería un equivalente oficial «el Espíritu Santo» $\rightarrow$ 圣灵 (shènglíng, el Espíritu Santo).

3) Traducción literal: consiste en traducir palabra por palabra un sintagma o expresión, y se puede combinar con el préstamo o el equivalente. Un ejemplo consistiría en traducir la obra de Lope de Vega Las ferias de Madrid por 《马德里集市》 (Mǎdélí Jíshì, Las ferias de / en Madrid).

4) Especificación: se añade información sobre el referente cultural. Esta categoría se subdivide en la de formulación completa y la de adición. La formulación completa consiste en completar abreviaciones, nombres oficiales o el nombre de alguien. Se opone a la condensación. Como ejemplos, cabría citar: «Ramón y Cajal» $\rightarrow$ (ShenYing) 圣地亚哥 · 拉蒙-卡哈尔 (Shèngdìyàgē Lāméng Kăhā'ěr, Santiago Ramón y Cajal). La adición consiste en añadir algún contenido semántico a través de merónimos, palabras polisémicas o hipónimos. Así, se pueden dar más detalles al espectador meta sobre el referente cultural origen. Por ejemplo, «Los fusilamientos» $\rightarrow$ 《枪杀起义者》 (Qiāngshā Qíyizhě, El fusilamiento de los rebeldes). Debido a que el referente pasa rápidamente en el diálogo y no se ve en la pantalla, la adición de «rebeldes», que es una palabra positiva en el contexto chino, ofrece más información al espectador meta que simplemente «fusilamientos» o la fecha de «el tres de mayo de 1808".

5) Amplificación: se refiere al uso de notas en la pantalla, las cuales suelen cobrar las dos formas siguientes (Wang 2017, 169) en la transferencia de los referentes culturales: a) notas insertas en la línea de los subtítulos en chino, al lado del objeto que se explica, entre corchetes y en tamaño más pequeño; b) notas libres en cualquier parte de la pantalla, normalmente con el mismo color que los subtítulos o con un color similar al fondo de la pantalla. La figura 1 muestra un ejemplo de notas insertas. En este caso el grupo de fansubbing ShenYing traduce «suflés" por 舒芙蕾 (shüfúlěi, suflés) mediante el equivalente oficial y el préstamo normalizado. Luego, al lado del referente traducido, pone una nota inserta entre corchetes y en tamaño pequeño (que hemos señalado con un recuadro rojo en la figura 1) que significa "dulce francés».

Peng $G_{A O}$

Cultura española en la pantalla china: la transferencia de referentes culturales...
CLINA

vol. 6-1, June 2020, 89-108

elSSN: 2444-1961

Ediciones Universidad de Salamanca - CC BY-NC-ND 


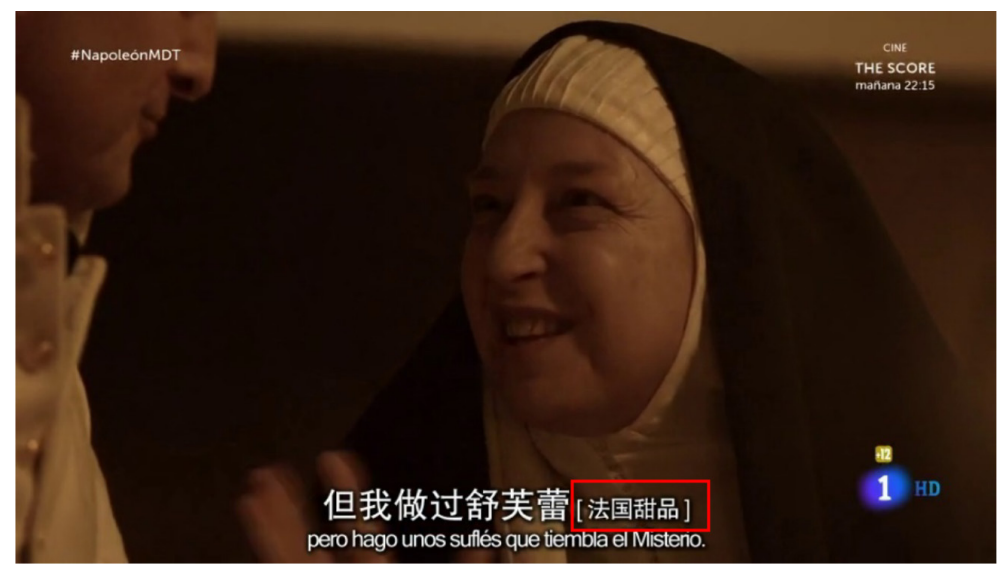

Figura 1. Nota en la pantalla sobre suflés. Episodio 12. ShenYing

6) Generalización: se altera el referente cultural por algún elemento más general, lo que se realiza a través del término genérico y la paráfrasis. Mediante el término genérico, el referente cultural del TO se permuta por un hiperónimo o un holónimo. Cabe citar el siguiente ejemplo: «números gordos» $\rightarrow$ (YYeTs) 彩票 (cǎipiào, lotería). La paráfrasis radica en cambiar el referente del TO por una frase más larga y general, pero con sentido similar, por ejemplo «libro de caballerías» $\rightarrow$ (YYeTs) 有关 骑士的书 (yǒuguān qíshì de shū, libro sobre caballeros).

7) Sustitución: incluye la sustitución cultural y la sustitución situacional. La sustitución cultural significa que el referente cultural del TO se permuta por otro referente, mientras que la sustitución situacional significa que el referente se elimina y se cambia por algún elemento que se corresponde con la situación. La sustitución cultural se realiza de dos maneras: con referentes transculturales y con referentes de la cultura meta. En la sustitución cultural por un referente transcultural, hay dos posibilidades. Una es sustituir el referente del TO por otro más conocido de la cultura origen para la audiencia meta; otra es sustituir el referente del TO por otro de una tercera cultura. A diferencia de la sustitución situacional, la generalización conserva una parte de su sentido, aunque se cambia el referente del TO por algo general. Al contrario, mediante la sustitución situacional se pierde completamente el sentido del referente del TO.

8) Creación discursiva: se crea una equivalencia efímera totalmente imprevisible fuera del TM. En nuestro corpus, se usa esta técnica en la traducción de apodos, productos de alimentación, títulos de obras literarias y de bellas artes, títulos de programas de televisión, etc. Cabe citar como ejemplo: «el Empecinado» $\rightarrow$ (YYeTs) 不屈者 (Bùqūzhě, Quien no se rinde).

9) Compensación: un elemento perdido en algún lugar de la traducción se compensa a través de una adición en otro lugar de dicha traducción. En nuestro estudio,

Peng $G_{A O}$

Cultura española en la pantalla china: la transferencia de referentes culturales... 
se dan la generalización y la especificación en la misma traducción. Citamos el siguiente ejemplo: «Vieja friendo huevos» $\rightarrow$ (ShenYing) 《煎鸡蛋的妇人》 (Jiān Jìdàn De Fùrén, Mujer friendo huevos de gallina).

10) Condensación: se suprime una parte del referente cultural del TO. Se usa normalmente en antropónimos y topónimos, y se opone a la formulación completa de la especificación. Como ejemplo, en algunos casos encontramos «Lope de Vega» $\rightarrow$ 维加 (Wéijiā, Vega). Si la condensación se realiza mediante acrónimos, acortamientos, abreviaturas o siglas, se denomina abreviación.

11) Omisión: se elimina el referente cultural del TO en el TM. Se utiliza frecuentemente en la transferencia de los referentes culturales no verbales.

\section{METODOLOGÍA}

En esta sección, presentaremos primero la serie El Ministerio del Tiempo y su distribución en China para introducir a continuación el corpus y la determinación concreta del nivel de transculturalidad y de centralidad de los referentes. Por último, hablaremos de las herramientas y el proceso de trabajo del presente estudio.

\subsection{El Ministerio del Tiempo y su distribución en China}

El Ministerio del Tiempo es una serie de ficción sobre la historia de España, creada por Pablo y Javier Olivares para RTVE y disponible en Netflix. Esta serie versa sobre un ministerio misterioso del gobierno, en el que los agentes deben hacer viajes en el tiempo a otras épocas de España para proteger a los personajes importantes y evitar cambios en la historia española. Además de los 34 episodios de las tres primeras temporadas, en 2020 comenzó a emitirse la $4^{a}$ temporada de la serie en la plataforma «A la carta» de RTVE.

Esta serie entró en China de manera no oficial gracias a grupos de fansubbing. El grupo ShenYing publicó el primer fansub de la serie en su foro en línea aproximadamente el 25 de septiembre de 2015. Después, en dos años terminó la traducción de los episodios emitidos de esta serie (hasta finales de 2017). En septiembre de 2016 el grupo YYeTs empezó también con la subtitulación de esta serie, pero a partir de mediados de 2017 dejó de publicar fansubs, por lo que su versión es incompleta: solo ha traducido la temporada 1, ocho episodios de la temporada 2 y un episodio de la temporada 3. Ambos fansubs disponen de dos líneas de subtítulos bilingües: el subtítulo en chino está arriba y el subtítulo en español está debajo.

En cuanto a la distribución oficial de la serie en China, las primeras dos temporadas se emitieron en el canal de pago Fengyun Juchang en febrero de 2018. Un año después, la plataforma de vídeos Bilibili compró la serie, así que, desde el 31 de marzo de 2019, las primeras dos temporadas están disponibles oficialmente en Bilibili.com.

Peng $G_{A O}$

Cultura española en la pantalla china: la transferencia de referentes culturales...
CLINA

vol. 6-1, June 2020, 89-108

elSSN: 2444-1961

Ediciones Universidad de Salamanca - CC BY-NC-ND 


\subsection{El corpus}

El corpus del presente estudio se compone de las versiones del grupo ShenYing y del grupo YYeTs de 5 episodios de la primera temporada, 4 episodios de la segunda temporada y 1 episodio de la tercera temporada. Los episodios fueron seleccionados por la disponibilidad de los fansubs, así como la abundancia y la variedad de referentes culturales. Puesto que cada episodio dura una hora aproximadamente, el análisis abarca 20 horas.

\subsection{Determinar el nivel de transculturalidad}

Como este parámetro de influencia se refiere a la familiaridad de los espectadores con respecto al TO y al TM, partimos del siguiente perfil del espectador origen y el espectador meta para determinar el nivel de transculturalidad de cada referente cultural en el corpus.

El espectador origen de la serie El Ministerio del Tiempo es muy amplio, dado que es una serie de televisión emitida en RTVE, una televisión pública en España y, además, está disponible en Netflix. En comparación con el espectador origen, el espectador meta de la misma serie es más específico, puesto que se refiere al espectador de los dos fansubs en chino. Partiendo de nuestras propias percepciones como fansubber y usuaria de plataformas de fansubbing durante años, en el presente estudio consideramos que el perfil del espectador meta de esta serie en China son personas de entre 16 y 40 años, la mayoría de ellas estudiantes de la lengua española o trabajadores en algún sector relacionado con español. En este sentido, son personas con conocimientos básicos sobre la cultura española. Asimismo, son aficionados al cine, a las series de televisión españolas o, al menos, a la cultura occidental. Por último, tienen una actitud abierta a conocimientos nuevos y quieren saber más sobre la cultura española. Siguiendo este perfil, se clasificaron, por ejemplo, «Edad de Oro» como referente transcultural, «el Hotel Ritz» como referente monocultural y «calle Grillo» como referente infracultural.

\subsection{Determinar el nivel de centralidad}

Teniendo en consideración el género de la serie (viajes en el tiempo a diferentes sitios), en el estudio se delimitó el nivel de centralidad según la tabla 1.

De acuerdo con la tabla 1, en diferentes episodios, el nivel del mismo referente puede ser diferente. Por ejemplo, «el Empecinado» del episodio 1 se clasificó como central a nivel macrotextual porque es el personaje principal de este episodio. Aun así, cuando se menciona rápidamente en el episodio 3 como el tema principal del episodio 1, se clasificó como secundario a nivel macro-, pero central a nivel microtextual, porque el episodio 3 versa sobre nazis y la reunión de Hendaya. Por la misma razón, «Lope de Vega» fue clasificado como central a nivel macrotextual en el episodio 2 y considerado 
como secundario a nivel macro-, pero central a nivel microtextual en el episodio 3, puesto que el episodio 2 trata de este escritor y su experiencia en la Armada Invencible.

Tabla 1. Determinación del nivel de centralidad

\begin{tabular}{|l|l|}
\hline Central a nivel macrotextual & $\begin{array}{l}\text { Relacionado con el tema principal de este episodio; } \\
\text { cambio de lugares y de épocas. }\end{array}$ \\
\hline $\begin{array}{l}\text { Secundario a nivel macro-, pero } \\
\text { central a nivel microtextual }\end{array}$ & $\begin{array}{l}\text { Relacionado con el tema principal de otro episodio o } \\
\text { que se va a mencionar más adelante en el mismo epi- } \\
\text { sodio; expresa humor e ironía; muestra el carácter de } \\
\text { los personajes. }\end{array}$ \\
\hline $\begin{array}{l}\text { Secundario a nivel macro- y nivel } \\
\text { microtextual }\end{array}$ & $\begin{array}{l}\text { Se mencionan muchos referentes en poco tiempo; el } \\
\text { diálogo no cambiará sin este referente; antropónimos y } \\
\text { topónimos en refranes o frases hechas. }\end{array}$ \\
\hline
\end{tabular}

\subsection{Herramientas y el proceso de trabajo}

Para el presente estudio, en vez de la recopilación automática de subtítulos a través de SubRip, se realizó la transcripción manual de los referentes culturales y los subtítulos relacionados mediante programas de Microsoft Office, debido a que ambos fansubs solo disponen de subtítulos «duros». Además, se utiliza el programa VLC para la visualización de los fansubs.

El proceso de trabajo de nuestro estudio se compone de seis pasos: en primer lugar, se identificaron y se recopilaron los referentes origen en los subtítulos en español, los referentes traducidos en los subtítulos en chino y las notas en la pantalla puestas en ambos fansubs. En segundo lugar, se creó un Excel con diferentes columnas que nos permitió recopilar de manera ordenada el contexto y la información complementaria que proporciona el canal no verbal cuando era necesario. Es decir, si el referente verbal aparece en la pantalla, cómo interactúan los personajes (incluye el diálogo y los gestos), etc. Este tipo de información sirve para determinar la centralidad de cada referente. En tercer lugar, se realizó la retraducción al español de los referentes traducidos y las notas en la pantalla en chino para ver la correspondencia del referente origen y el referente traducido. En cuarto lugar, se clasificaron los referentes origen y las técnicas de traducción utilizadas por los dos grupos de fansubbing de acuerdo con el marco teórico. Asimismo, se estableció la categoría de referentes no verbales y se repitieron los cuatro pasos anteriores. Por último, se analizaron las relaciones entre la clasificación de referentes culturales y la clasificación de técnicas de traducción.

\section{RESULTADOS Y ANÁLISIS}

En el corpus se encontraron 729 referentes culturales en los 10 episodios seleccionados. Entre ellos, existen 649 referentes verbales (89\%) y 80 referentes no verbales 
(11\%). A continuación, se presentarán los referentes culturales encontrados de acuerdo con los dominios, la transculturalidad y la centralidad. Luego, se mostrarán las técnicas de traducción encontradas en el estudio y se analizarán las relaciones entre los referentes culturales y las técnicas de traducción.

\subsection{Los dominios de los referentes culturales del corpus}

En cuanto a los dominios de referentes culturales, se encontraron 27 dominios en la categoría de referentes verbales y 18 dominios en la categoría de referentes no verbales. La tabla 2 muestra el porcentaje y los ejemplos de los dominios encontrados en el corpus. En la categoría de referentes verbales, la ecología, educación, el derecho y las marcas comerciales ocupan menos del $1 \%$. Por consiguiente, no se muestran sus ejemplos en la tabla.

Tabla 2. Distribución de los dominios de referentes culturales en el corpus

\begin{tabular}{|c|c|}
\hline \multicolumn{2}{|c|}{637 referentes culturales verbales ${ }^{2}$} \\
\hline Dominios & Ejemplos \\
\hline Antropónimos (28%) & Velázquez \\
\hline Historia (20 \%) & La Guerra de Cuba \\
\hline Topónimos (17%) & Hendaya \\
\hline Literatura (14 \%) & Nanas de la cebolla \\
\hline Religión (13 \%) & la Inquisición \\
\hline Entretenimiento (10 \%) & Richard Kimble \\
\hline Bellas artes (8 \%) & Las meninas \\
\hline Nombres institucionales (6 \%) & El MoMA \\
\hline Gobierno (4 \%) & la Guardia Civil \\
\hline $\begin{array}{l}\text { Apodos; alimentación; costumbres; deportes; } \\
\text { economía; música; objetos materiales (3 \%) }\end{array}$ & $\begin{array}{l}\text { El Empecinado; patatas bravas; la misa del } \\
\text { Gallo; Naranjito }\end{array}$ \\
\hline Gentilicio; Títulos profesionales (2 \%) & La Tizona; los municipales \\
\hline $\begin{array}{l}\text { Acontecimientos contemporáneos; ciencia } \\
\text { y tecnología; festividades; medios de } \\
\text { comunicación; títulos nobiliarios (1 \%) }\end{array}$ & 11-M; WhatsApp; la Noche de Difuntos \\
\hline $\begin{array}{l}\text { Ecología; educación; derecho; marcas } \\
\text { comerciales (<1\%) }\end{array}$ & \\
\hline
\end{tabular}

2. 12 referentes son excluidos del análisis por la falta de un fragmento en la versión del grupo YYeTs del episodio 2.

Peng GAO

Cultura española en la pantalla china: la transferencia de referentes culturales... 


\begin{tabular}{|c|c|}
\hline \multicolumn{2}{|c|}{80 referentes culturales no verbales } \\
\hline Dominios & Ejemplos \\
\hline Costumbres (30 \%) & Besar la mano \\
\hline Historia (20 \%) & Pluma de ave \\
\hline Objetos materiales (14 \%) & Cafetera tradicional \\
\hline Alimentación; bellas artes; religión (13%) & $\begin{array}{l}\text { Acueducto de Segovia; hacer la señal de la } \\
\text { cruz }\end{array}$ \\
\hline Entretenimiento (8 \%) & Saludo vulcano del señor Spock \\
\hline Antropónimos (6 \%) & Imagen de la Reina Letizia en la televisión \\
\hline Nombres institucionales; topónimos (4 \%) & $\begin{array}{l}\text { Se ve la fachada con cristales del Museo } \\
\text { Nacional Centro de Arte Reina Sofía }\end{array}$ \\
\hline $\begin{array}{l}\text { Acontecimientos contemporáneos; economía; } \\
\text { música (3 \%) }\end{array}$ & $\begin{array}{l}\text { Celebraciones del primer aniversario del Rey } \\
\text { Felipe como jefe del Estado }\end{array}$ \\
\hline $\begin{array}{l}\text { Ciencia y tecnología; deportes; festividades; } \\
\text { gobierno; títulos nobiliarios (1\%) }\end{array}$ & $\begin{array}{l}\text { Un jugador del Atleti levanta la Copa del Rey; } \\
\text { se ve a Adolfo Suárez en la televisión de una } \\
\text { habitación }\end{array}$ \\
\hline
\end{tabular}

Un referente cultural puede pertenecer a dos dominios. Además de ser un antropónimo, «Richard Kimball» también pertenece al dominio del entretenimiento, porque es un personaje de la serie de televisión estadounidense El fugitivo (1963). Por la misma razón, el referente «Velázquez» pertenece al dominio de antropónimos y al de las bellas artes. Este tipo de referentes culturales se consideran referentes multifuncionales $(\mathrm{Pe}$ dersen 2011, 60). Asimismo, también hay casos en los que un referente cultural está incrustado o integrado (Pedersen 2011, 60) en otro referente. En el referente «Reina Letizia», por ejemplo, se combinan el título nobiliario «reina» y el antropónimo «Letizia».

\subsection{Nivel de transculturalidad y de centralidad de referentes culturales del corpus}

La figura 2 muestra la distribución de los referentes transculturales, monoculturales e infraculturales, o sea, el nivel de transculturalidad de los referentes culturales en el corpus.

Teniendo en cuenta el perfil del espectador meta, el $48 \%$ de los referentes verbales del corpus fueron clasificados como referentes transculturales. Así pues, «los Reyes Católicos» y «la Guerra Civil» pertenecen al dominio de la historia; «las Ramblas» y «la Puerta del Sol», al de los topónimos. En la categoría de referentes no verbales, el 64 \% se consideraron referentes transculturales. En esta categoría, los referentes transculturales más comunes se sitúan en costumbres, historia y productos de alimentación, como, por ejemplo, «hacer la señal de la cruz», "dar besos para saludar» y «tortilla de patatas».

Peng GAO

Cultura española en la pantalla china: la transferencia de referentes culturales... 
Como es probable que el espectador meta no conozca con la misma profundidad que el espectador origen ni la geografía del mundo hispanohablante, los escritores españoles o sus obras, ni las formas de entretenimiento habituales en España, una gran parte de referentes verbales (45\%) fueron clasificados como monoculturales. Cabe citar los ejemplos de "Atapuerca» entre los topónimos, «La dama boba» y "Jovellanos» en el dominio de la literatura y «el mus» en el área de entretenimiento. En cuanto a los referentes no verbales, uno de los ejemplos de referentes monoculturales puede ser el discurso de dimisión de "Adolfo Suárez». Aunque su nombre resulta suficientemente conocido por el espectador meta en China gracias a su papel en la transición, es difícil para el espectador chino identificar a este político y su discurso en 1981 solamente con la información del canal no verbal. Por último, el $7 \%$ de los referentes verbales y el $7 \%$ de los referentes no verbales fueron clasificados como infraculturales. Destacamos, por ejemplo, «la Santa Hermandad» en la categoría de gobierno y de historia, «el chapín» entre los objetos materiales y de historia, así como el programa de televisión Más allá - Casas encantadas en el área del entretenimiento.

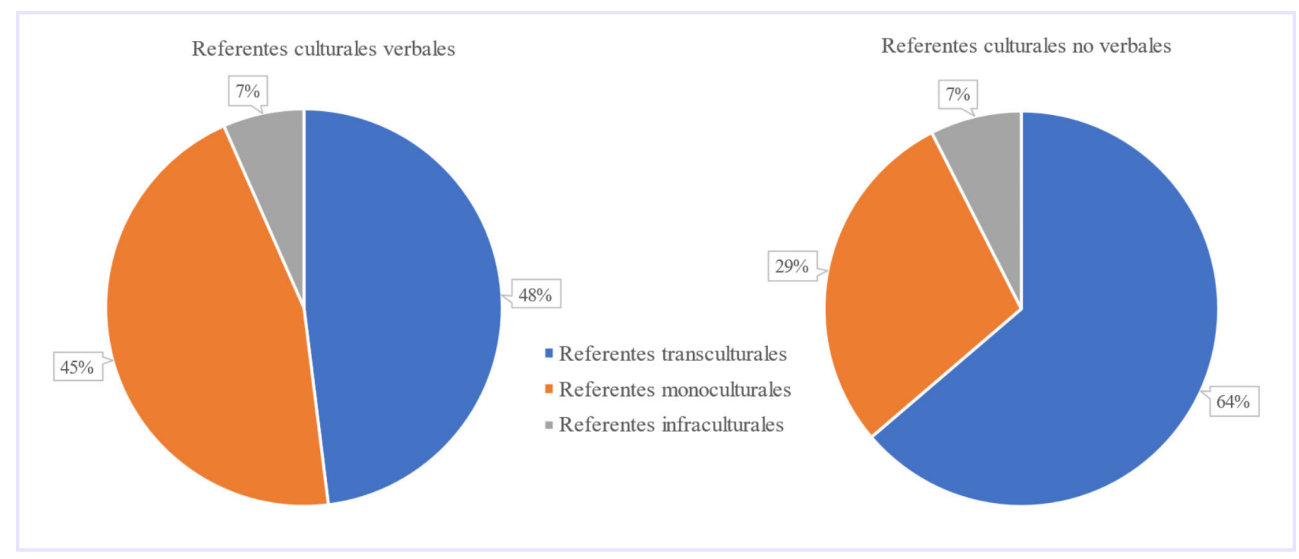

Figura 2. Nivel de transculturalidad de referentes culturales

Después de la transculturalidad, nos vamos a centrar en el nivel de centralidad de los referentes culturales del corpus. Como se ha mencionado antes, de acuerdo con el nivel de alta o baja incidencia de este parámetro, los referentes culturales se subdividen en tres tipos: central a nivel macrotextual; secundario a nivel macro-, pero central al nivel microtextual; y secundario a nivel macro- y nivel microtextual.

La figura 3 muestra la distribución de los referentes culturales del corpus según el nivel de centralidad. Los referentes que son centrales a nivel macrotextual son una proporción reducida en comparación con los restantes dos tipos de referentes. Esto se debe a los criterios establecidos en el subapartado 3.4. Además, en la categoría de los referentes verbales, los referentes que son secundarios a nivel macro-, pero centrales

Peng $\mathrm{G}_{A O}$

Cultura española en la pantalla china: la transferencia de referentes culturales... 
a nivel microtextual son muy frecuentes (46\%), mientras que, en la categoría de los referentes culturales no verbales, los referentes más habituales son los secundarios a nivel macro- y nivel microtextual (73\%).

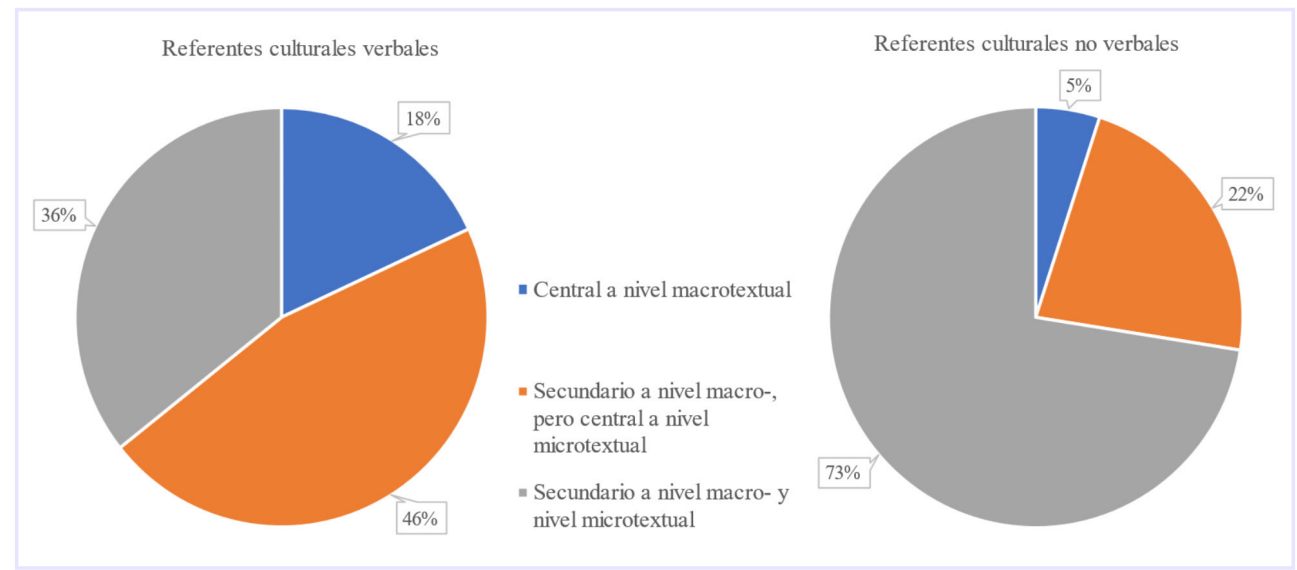

Figura 3. Nivel de centralidad de referentes culturales

Entre los referentes no verbales, existe una multitud de detalles ligados a un sitio o un periodo específico. Así podemos mencionar la escultura del ángel del Edificio Metrópolis de Madrid en el episodio 12 y los camareros vestidos con la ropa tradicional del País Vasco en el episodio 22. Estos referentes no forman parte del tema principal del episodio. Tampoco son relevantes para el argumento o el contexto de la serie. Además, algunos de ellos son tan específicos como, por ejemplo, la fachada del monasterio de Santa Clara que aparece en el episodio 12, que posiblemente ni el espectador origen sepa identificar. Estos elementos se introducen en la serie para crear una atmósfera especial con el fin de que los espectadores puedan «viajar a otras épocas» con los personajes. Este tipo de referentes no verbales se pueden denominar «referentes asincrónicos» (Ranzato 2013, 91).

\subsection{Técnicas de traducción en el corpus}

En esta sección presentaremos, en primer lugar, la distribución de las técnicas de traducción utilizadas en la transferencia de los referentes culturales del corpus. A continuación, analizaremos la relación entre la clasificación de los referentes culturales y la de las técnicas de traducción para responder a la pregunta: entre los dominios, la transculturalidad y la centralidad, ¿cuáles son los factores más importantes en la transferencia de los referentes culturales en el fansubbing del español al chino?

Peng $\mathrm{G}_{A O}$

Cultura española en la pantalla china: la transferencia de referentes culturales... 


\subsubsection{Referentes verbales}

La tabla 3 muestra la distribución de cada subcategoría de las técnicas de traducción utilizadas en el corpus. Como se puede apreciar, no hay mucha diferencia entre la versión del grupo ShenYing y la del grupo YYeTs salvo en la categoría de la amplificación.

Tabla 3. Distribución de técnicas de traducción de referentes verbales

\begin{tabular}{|c|c|c|c|c|c|}
\hline Técnicas de traducción & SY & YY & Técnicas de traducción & SY & YY \\
\hline Equivalente oficial & $31,7 \%$ & $30,0 \%$ & Creación discursiva & $1,9 \%$ & $2,3 \%$ \\
\hline Préstamo normalizado & $20,3 \%$ & $19,9 \%$ & Omisión & $1,7 \%$ & $2,5 \%$ \\
\hline Traducción literal & \multicolumn{2}{|c|}{$7,7 \%$} & Condensación & $1,5 \%$ & $1,7 \%$ \\
\hline Préstamo no normalizado & $7,5 \%$ & $6,5 \%$ & Sustitución cultural & $1,3 \%$ & $1,9 \%$ \\
\hline Equivalente acuñado & $7,2 \%$ & $6,5 \%$ & Paráfrasis & $1,1 \%$ & $1,3 \%$ \\
\hline Amplificación & $6,0 \%$ & $8,2 \%$ & Abreviación & \multicolumn{2}{|c|}{$0,8 \%$} \\
\hline Término genérico & $4,1 \%$ & $4,4 \%$ & \multirow{3}{*}{$\begin{array}{l}\text { Sustitución situacional, } \\
\text { préstamo completo y } \\
\text { compensación }\end{array}$} & \multirow{3}{*}{\multicolumn{2}{|c|}{$\leq 0,7 \%$}} \\
\hline Adición & $3,4 \%$ & $2,3 \%$ & & & \\
\hline Formulación completa & $2,9 \%$ & $3,0 \%$ & & & \\
\hline
\end{tabular}

Por un lado, las técnicas más frecuentemente utilizadas son el equivalente oficial y el préstamo normalizado. Esta preferencia se debe a la abundancia de antropónimos y topónimos en el corpus. Por otro lado, raramente se utilizan la sustitución situacional (4 en el fansub de ShenYing y 6 en el de YYeTs), el préstamo completo (3 en el fansub de ShenYing y 3 en el de YYeTs) y la compensación (1 en el fansub de ShenYing). Asimismo, se identificaron 20 casos más de amplificación en la versión de YYeTs que en la de ShenYing.

Con el fin de responder a la pregunta formulada anteriormente, se analizaron y se compararon los datos de ambos grupos para comprender mejor el uso de cada técnica. La tabla 4 muestra la importancia de los dominios, la transculturalidad y la centralidad de los referentes culturales en el uso de cada técnica de traducción. El símbolo «+ +» representa «muy importante», «+» significa «importante» mientras que el signo «-» representa «poco importante» $y$ « $x$ » significa «sin importancia». En la tabla, las líneas 17) y 18) aparecen incompletas debido a la falta de datos. Por limitaciones de espacio, no analizamos (os puntos 1), 2), 4), 5) y de 16) a 18).

Peng $G_{A O}$

Cultura española en la pantalla china: la transferencia de referentes culturales... 
Tabla 4. Importancia de los dominios, la transculturalidad y la centralidad

\begin{tabular}{|c|c|c|c|c|}
\hline \multicolumn{2}{|c|}{ Técnicas de traducción } & Dominios & Transculturalidad & Centralidad \\
\hline \multicolumn{2}{|l|}{ 1) Equivalente oficial } & ++ & ++ & $x$ \\
\hline \multicolumn{2}{|l|}{ 2) Préstamo normalizado } & ++ & + & ++ \\
\hline \multicolumn{2}{|l|}{ 3) Traducción literal } & - & ++ & + \\
\hline \multicolumn{2}{|l|}{ 4) Préstamo no normalizado } & ++ & + & $x$ \\
\hline \multicolumn{2}{|l|}{ 5) Equivalente acuñado } & + & ++ & + \\
\hline \multirow{2}{*}{ 6) Amplificación } & SY & + & ++ & ++ \\
\hline & YY & ++ & ++ & - \\
\hline \multicolumn{2}{|l|}{ 7) Término genérico } & + & + & ++ \\
\hline \multicolumn{2}{|l|}{ 8) Adición } & + & ++ & ++ \\
\hline \multicolumn{2}{|l|}{ 9) Formulación completa } & ++ & $x$ & + \\
\hline \multicolumn{2}{|l|}{ 10) Creación discursiva } & ++ & ++ & - \\
\hline \multicolumn{2}{|l|}{ 11) Omisión } & $x$ & + & ++ \\
\hline \multicolumn{2}{|l|}{ 12) Condensación } & ++ & ++ & + \\
\hline \multicolumn{2}{|l|}{ 13) Sustitución cultural } & ++ & ++ & + \\
\hline \multicolumn{2}{|l|}{ 14) Paráfrasis } & - & + & ++ \\
\hline \multicolumn{2}{|l|}{ 15) Abreviación } & ++ & ++ & $x$ \\
\hline \multirow{2}{*}{ 16) Sustitución situacional } & SY & ++ & ++ & - \\
\hline & YY & - & ++ & ++ \\
\hline \multicolumn{2}{|l|}{ 17) Préstamo completo } & ++ & \multicolumn{2}{|c|}{ (Faltan datos) } \\
\hline \multicolumn{2}{|l|}{ 18) Compensación } & \multicolumn{3}{|c|}{ (Faltan datos) } \\
\hline
\end{tabular}

Traducción literal: en cuanto a la traducción literal, se puede explicar desde dos perspectivas. Por una parte, entre los tres factores, la transculturalidad tiene mayor importancia en el uso de esta técnica. Según nuestros datos, los fansubbers la prefieren cuando los referentes son poco conocidos en la cultura china, dado que en esta técnica los referentes monoculturales e infraculturales representan el 68,1\%. Por ejemplo, «la Guerra de Cuba» se traduce por 古巴战争 (Gǔbā Zhànzhēng, Guerra de Cuba), aunque su equivalente oficial es 美西战争 (Měi-Xī Zhànzhēng, Guerra Hispano-Estadounidense); el referente «las pinturas negras» de Goya se traduce por 黑色 绘画 (hēisè huihuà, las pinturas negras) y Poeta en Nueva York se traduce por 《诗人 在纽约》 (Shĩrén Zài Niǔyuē, Poeta en Nueva York). Por otra parte, es probable que

Peng $G_{A O}$

Cultura española en la pantalla china: la transferencia de referentes culturales... 
Ios fansubbers utilicen esta técnica por la transparencia del referente origen. Es decir, cuando el referente origen se compone de palabras inteligibles, la traducción literal se puede entender sin problemas en la cultura meta.

Amplificación: en el análisis se observaron diferencias de uso de esta técnica entre el fansub de ShenYing y el fansub de YYeTs. Aunque la transculturalidad tiene la misma importancia en los dos fansubs (véase la tabla 4), ShenYing prefiere esta técnica para los referentes monoculturales e infraculturales, mientras que YYeTs lo usa más en los referentes transculturales e infraculturales. Asimismo, ShenYing hace hincapié en la centralidad y no usa mucho esta técnica en los referentes que son secundarios a nivel macro- y nivel microtextual. En cambio, YYeTs pone notas para antropónimos o topónimos que no tienen importancia en el argumento. En el episodio 1 se menciona el «11-M», conocido para el espectador meta en China, y cuya función es presentar la experiencia del personaje como enfermero, por lo que se clasificó como secundario a nivel macro- y nivel microtextual. Aun así, YYeTs pone una nota en la pantalla: 11-M 于 2004年3月11日在马德里发生的一系列恐怖袭击 (11-M: una serie de atentados terroristas que ocurrieron el 11 de marzo de 2004 en Madrid).

Término genérico: la centralidad es más importante para entender las frecuencias de uso de esta técnica, ya que el 54,1 \% de referentes culturales traducidos con ella son secundarios a nivel macro- y nivel microtextual. Así pues, el «11-M» se convierte en 311事件 (Sānyīyī Shijiàn, el acontecimiento 311) en la versión del grupo ShenYing del primer episodio de la serie.

Adición y formulación completa: numerosos referentes monoculturales, infraculturales y los referentes que no son centrales a nivel macrotextual se traducen mediante la adición. Citamos a título de ejemplo: «el Rosellón» $\rightarrow$ (ShenYing) 鲁西荣争夺战 (Lǔxiróng Zhēngduózhàn, Guerra de Disputa de Rosellón); «Flandes» $\rightarrow$ (YYeTs) 佛兰德 斯的战役 (Fólándésĩ de zhànyì, batalla de Flandes). Además, se usa esta técnica en los antropónimos que son centrales a nivel macrotextual, como por ejemplo en, «los Reyes Católicos» $\rightarrow$ 天主教双王时期 / 时代 (Tiānzhǔjiào Shuāngwáng shíqi / shídài, la época de los dos Reyes Católicos). En comparación con la adición, en la formulación completa son más importantes los topónimos (que ocupan el 42,3 \%) y los nombres institucionales (con un 26,9%). Valga a modo de ejemplo «Sicilia» $\rightarrow$ 西西里岛 (Xixî̃ Dăo, la isla de Sicilia), que en esta traducción al chino coincide con el equivalente oficial.

Creación discursiva: se utiliza normalmente en los referentes monoculturales de apodos, literatura, música, topónimos y entretenimiento como, por ejemplo, La dama boba $\rightarrow$ (ShenYing) 《木头姑娘》 (Mùtou Güniang, La chica de madera); Adeste fideles $\rightarrow$ (ShenYing) 《真挚来临》 (Zhēnzhi Láilín, Llega la sinceridad).

Omisión: se usa con frecuencia en las referencias indirectas que son monoculturales y secundarias a nivel macro- y nivel microtextual. El episodio 5, por ejemplo, incorpora un fragmento de Champú de huevo de Tino Casal, pero no se menciona el título de esta canción directamente en el diálogo. En este caso solamente se traduce la letra en los subtítulos en chino. Además, esta técnica también se usa mucho para

Peng $\mathrm{G}_{A O}$

Cultura española en la pantalla china: la transferencia de referentes culturales...
CLINA

vol. 6-1, June 2020, 89-108

elSSN: 2444-1961

Ediciones Universidad de Salamanca - CC BY-NC-ND 
los referentes secundarios a nivel macro- y nivel microtextual que solo aparecen en el canal visual.

Condensación: se utiliza normalmente en los referentes transculturales del dominio de los antropónimos y la historia. Como ejemplo cabe citar «Salvador Dalíı» $\rightarrow$ 达利 (Dáli, Dalî) y «Segunda Guerra Mundial» $\rightarrow$ 二战 (Ėzhàn, Segunda Guerra).

Sustitución cultural: los referentes monoculturales o infraculturales de alimentación y entretenimiento suelen traducirse con esta técnica. Así pues, ShenYing y YYeTs traducen Sauerkraut por 酸菜 (suāncài, verduras agrias), que es similar al chucrut, pero está hecho con diferentes verduras; «merendar» por 吃/喝下午茶 (chī / hē xiàwǔchá, tomar té de tarde), que se considera como un símbolo de la cultura inglesa en China y difiere de la forma de merendar de España.

Paráfrasis: todos los referentes traducidos con esta técnica son secundarios a nivel macro-, pero centrales a nivel microtextual y el 60 \% de ellos son monoculturales. La paráfrasis depende mucho del contexto, por lo que el mismo referente puede ser traducido de diferentes maneras. Así pues, en el episodio 2, dos personajes que fueron antes a una taberna del siglo XVI ahora llegan a una fonda barata para buscar a un hombre. Al entrar, un personaje afirma que «la Taberna del Gallego comparada con esto es el Ritz». En este caso, ShenYing traduce «el Ritz» por 豪华 (háohuá, lujoso). No obstante, en el siguiente episodio, cuando este referente aparece en la pantalla, se traduce por 丽兹酒店 (Lizi Jiǔdiàn, Hotel Ritz).

Abreviación: se usa frecuentemente en los nombres institucionales transculturales (el 71,4\%). Como ejemplo se pueden citar los siguientes: «el Madrid» $\rightarrow$ 皇马 (Huáng Mă, MAD, abreviación del Real Madrid); «el Banco Central Europeo» $\rightarrow$ 欧洲央 行 (Ōuzhōu-Yāngháng, BCE, abreviación del Banco Central Europeo).

En resumen, vemos que en los referentes verbales los tres factores inciden en el siguiente orden de prelación: la transculturalidad $>$ los dominios $>$ la centralidad.

\subsubsection{Referentes culturales no verbales}

La omisión es la técnica más frecuentemente utilizada en la transferencia de los referentes no verbales. En los 80 referentes no verbales del corpus, se encuentra solo un caso de amplificación en el fansub del grupo ShenYing.

El único caso de amplificación se da en relación con una tapa. En el episodio 5, un personaje (Julián) se encuentra con sus padres en un bar y piden algunas bebidas. Un rato después, el dueño del bar les pone cacahuetes como tapa (véase la figura 4). Entonces, el padre de Julián mira al dueño y se queja: «la crisis... Ya podías poner algunas gambitas». Al lado de esta frase, el grupo ShenYing pone una nota en la pantalla: 西班牙酒吧里一般随酒水附赠小吃 (en los bares de España, normalmente se regalan tapas con las bebidas alcohólicas y refrescos). En este caso, el grupo ShenYing intenta explicar con esta nota en la pantalla la costumbre de tomar tapas acompañando a las cervezas o al vino en los bares españoles.

Peng $\mathrm{G}_{A O}$

Cultura española en la pantalla china: la transferencia de referentes culturales... 


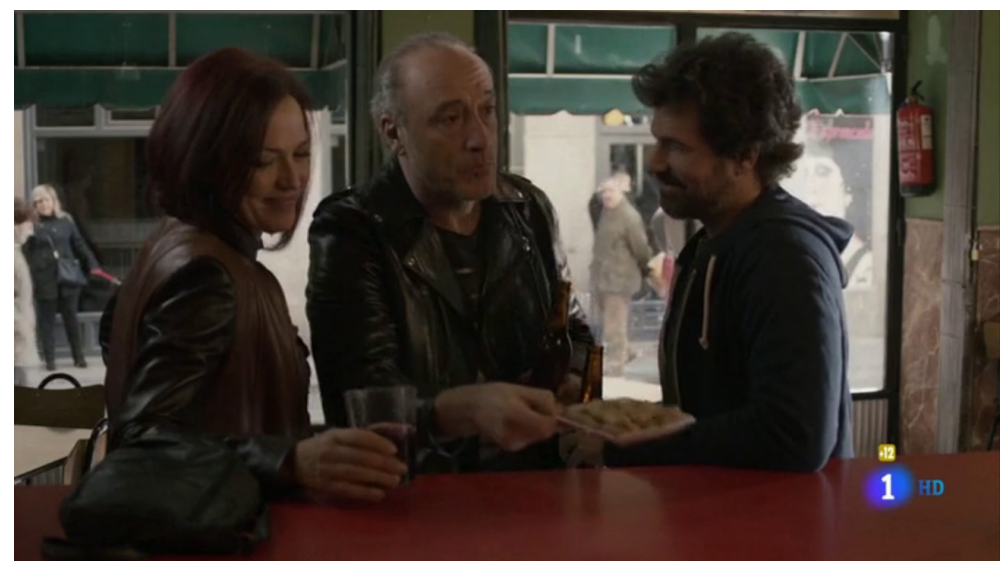

Figura 4. Una tapa de cacahuetes. Episodio 5. ShenYing

\section{CONCLUSIONES}

El presente artículo estudia el fenómeno del fansubbing del español al chino haciendo hincapié en la transferencia de los referentes culturales. Para ello, procede a analizarlos con la ayuda de una clasificación propia, establecida a partir de las propuestas más conocidas en el ámbito de los Estudios de Traducción. Si bien se trata de un corpus limitado a una serie de televisión y a un género muy particular, los resultados parecen extrapolables a otros casos.

Los resultados encontrados nos muestran la transferencia de los referentes culturales en el fansubbing del español al chino y responden a la pregunta de qué factor es más importante en esta transferencia (los dominios, la transculturalidad o la centralidad). En la categoría de referentes verbales, la transculturalidad aparece como el factor más influyente. Además, los dominios también tienen peso importante, especialmente en relación con algunas técnicas como la del equivalente y la del préstamo. El nivel de transculturalidad y los dominios interactúan e influyen conjuntamente en la selección de las técnicas de traducción de los referentes verbales. En cambio, la influencia de la centralidad es mucho menor en comparación con los dos factores restantes. La situación es diferente en los referentes no verbales. En esta categoría, la omisión es la técnica de traducción más utilizada y no se observa la prelación de ninguno de los tres factores. Asimismo, los resultados también señalan la necesidad de elaborar una clasificación de los referentes culturales tanto según sus características inherentes (los dominios) y la relación entre el TO y el TM (la transculturalidad) como según la importancia del propio referente en el argumento (la centralidad) y su papel en los textos audiovisuales (verbales o no verbales).

Peng $\mathrm{G}_{A O}$

Cultura española en la pantalla china: la transferencia de referentes culturales...
CLINA

vol. 6-1, June 2020, 89-108

elSSN: 2444-1961

Ediciones Universidad de Salamanca - CC BY-NC-ND 
Con respecto a las limitaciones del presente estudio, admitimos que existe cierta subjetividad en la determinación del nivel de transculturalidad y de centralidad de los referentes culturales, porque no siempre es clara la línea divisoria entre diferentes niveles. Teniendo en consideración esta limitación, en el futuro sería interesante llevar a cabo algunos estudios de recepción y de comparación, por ejemplo con los danmu de la versión oficial disponible en Bilibili (sobre este tema, véase Zhang y Cassany 2019), con el fin de mejorar la determinación del nivel de transculturalidad y el de centralidad de cada referente cultural y, así, perfeccionar el modelo de análisis utilizado en el presente estudio y profundizar en el análisis de todas las técnicas de traducción identificadas en la transferencia de referentes culturales en el fansubbing del español al chino.

\section{BIBLIOGRAFÍA}

CASAS-Tost, Helena y Jinyang Cul. 2019. «El tiempo entre costuras: análisis contrastivo entre la subtitulación profesional y la del fansubbing al chino». Sendebar 30: 11-34.

Cul, Jinyang. 2017. El fenómeno del fansubbing en China: análisis comparativo entre la versión de fansubs y la profesional de la serie española El tiempo entre costuras. Trabajo Fin de Máster. Universitat Autònoma de Barcelona.

Díaz-Cintas, Jorge y Pablo Muñoz SÁnchez. 2006. «Fansubs: Audiovisual Translation in an Amateur Environment». The Journal of Specialised Translation 6: 37-52. Acceso el 13 de octubre de 2018. https://www.jostrans.org/issue06/art_diaz_munoz.pdf

Díaz-CInTAS, Jorge y Aline Remael. 2007. Audiovisual Translation: Subtitling. Manchester: St Jerome.

FERRER Simó, María Rosario. 2005. «Fansubs y scanlations: la influencia de los aficionados en los criterios profesionales». Puentes 6: 27-44.

Hurtado AlbiR, Amparo. 2001. Traducción y traductología: introducción a la traductología. Madrid: Cátedra.

LEE, Yvonne. 2018. «Non-professional Subtitling». En The Routledge Handbook of Chinese Translation, editado por Chris Shei y Zhao-Ming Gao, 566-79. Londres y Nueva York: Routledge.

Lv, Yuyong y Min Lı. 2015. "On the New Features of Online Film and TV Subtitle Translation in China». International Journal of English Linguistics 5 (6): 122-127.

MolinA, Lucía. 2006. El otoño del pingüino: análisis descriptivo de la traducción de los culturemas. Castelló de la Plana: Publicacions de la Universitat Jaume I, D.L.

NedergaARd-Larsen, Birgit. 1993. "Culture-bound Problems in Subtitling». Perspectives: Studies in Translatology 1 (2): 207-40.

Pedersen, Jan. 2011. Subtitling Norms for Television: an exploration focussing on extralinguistic cultural references. Ámsterdam y Filadelfia: John Benjamins Publishing.

Pérez-GonzÁlez, Luis y Şebnem Susam-SARAEVA. 2012. «Non-professionals Translating and Interpreting». The Translator 18 (2): 149-65.

PYM, Anthony. 2000. Negotiating the Frontiers: Translators and Intercultures in Hispanic History. Manchester: St. Jerome.

RANZATO, IRENE. 2013. The Translation of Cultural References in the Italian Dubbing of Television Series. Tesis Doctoral. Imperial College London. 
SANTAMARIA, Laura. 2001. Subtitulació i referents culturals. La traducció com a mitjà d'adquisició de representacions mentals. Tesis Doctoral. Universitat Autònoma de Barcelona.

TIAN, Yuan. 2011. Fansub Cyber Culture in China. Trabajo Fin de Máster. Georgetown University. WANG, Dingkun. 2017. «Fansubbing in China - With Reference to the Fansubbing Group YYeTs». The Journal of Specialised Translation 28: 165-90. Acceso el 12 de febrero de 2020. https:// www.jostrans.org/issue28/art_wang.php

WANG, Dingkun y Xiaochun ZHANG. 2016. «ldeological Manipulation of Controversial Information: The Unusual Case of the Chinese-Subtitled Version of House of Cards». Altre Modernità, n extraordinario 1: 1-20.

YYETs. 2009. “ (转) 中国字幕组以及 YYeTs 字幕组的历史简介 [(compartido) Introducción breve de la historia de grupos de fansubbing de China y del grupo de fansubbing YYeTs]». Acceso el 16 de febrero de 2020. https://www.douban.com/note/131650274/

ZABALBEASCOA, Patrick. 2001. «El texto audiovisual: factores semióticos y traducción». En jDoble o Nada!, editado por John D. Sanderson, 113-26. Alicante: Editorial Universitat d' Alacant.

ZHANG, Letizia-Tian y Daniel CASSANy. 2019. «El fenómeno «danmu» y la participación mediática: Comprensión intercultural y aprendizaje de lenguas a través de «El Ministerio del Tiempo»». Comunicar 27 (58): 19-29.

ZHANG, Letizia-Tian y Daniel CASSANY. 2016. «Fansubbing del español al chino: organización, roles y normas en la escritura colaborativa». BiD: textos universitaris de biblioteconomia $i$ documentació 37: 1-12. Acceso el 16 de febrero de 2020. http://bid.ub.edu/es/37/tian.htm 